\title{
Article \\ Hybrid Algorithm for Anomaly Removal in Time Series Data Mining
}

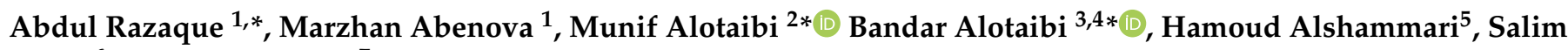 \\ Hariri $^{6}$ and Aziz Alotaibi ${ }^{7}$
}

1 Department of Computer Engineering and Information Security, International Information Technology University, Kazakhstan; a.razaque@iitu.edu.kz

2 Department of Computer Science, Shaqra University, Shaqra 15526, Saudi Arabia; munif@su.edu.sa

3 Sensor Networks and Cellular Systems (SNCS) Research Center, University of Tabuk, Tabuk 47731, Saudi Arabia

4 Department of Information Technology, University of Tabuk, Tabuk 47731, Saudi Arabia; b-alotaibi@ut.edu.sa

5 Computer and Information Science College, Jouf University, Jouf, Saudi Arabia

6 Department of Electical and Computer Engineering, University of Arizona, Tucson, Arizona 85721

7 Computers and Information Technology College, Taif University, Taif, Saudi Arabia

* Correspondence: a.razaque@iitu.edu.kz; munif@su.edu.sa; b-alotaibi@ut.edu.sa

\begin{abstract}
Time series data are significant and are derived from temporal data, which involve real numbers representing values collected regularly over time. Time series have a great impact on many types of data. However, time series have anomalies. We introduce hybrid algorithm named novel matrix profile (NMP) to solve the all-pairs similarity search problem for time series data. The proposed NMP inherits the features from two state-of-the art algorithms: similarity timeseries automatic multivariate prediction (STAMP), and short text online microblogging protocol (STOMP). The proposed algorithm caches the output in an easy-to-access fashion for single- and multidimensional data. The proposed NMP algorithm can be used on large data sets and generates approximate solutions of high quality in a reasonable time. The proposed NMP can also handle several data mining tasks. It is implemented on a Python platform. To determine its effectiveness, it is compared with the state-of-the-art matrix profile algorithms i.e., STAMP and STOMP. The results confirm that the proposed NMP provides higher accuracy than the compared algorithms.
\end{abstract}

Keywords: Time series; NMP algorithm; anomalies; data mining; similarities in time series; clustering

\section{Introduction}

Data mining is the analysis of (often large) observational data sets to find unsuspected relationships and to summarize the data in novel ways that are both understandable and useful to the data owner [1]. The relationships and summaries derived from a data mining exercise are often referred to as models or patterns (e.g., linear equations, laws, clusters, tables, tree structures, and repeated patterns of time series [2,3]). Data mining professionals tend to use fast, unsupervised methods in the early stages of the data mining process. Thus, data mining tasks (e.g., motif discovery, discord discovery, clustering, and segmentation) should be handled for time series data [4-8]. However, time series have anomalies due to similarities $[9,10]$. The scalable and sturdy similarity join algorithm has been proposed for handling time series anomalies [11]. However, it has a number of issues in the text domain, including community discovery, duplicate detection, collaborative filtering, clustering, and refinement of queries. Almost all of the similarity-handling algorithms for time series data either solve these problems partially or fail to address the issues [12]. A novel LSH-based method has been introduced for similarity time-series automatic multivariate prediction (STAMP) [13]. It is based on locality-sensitive hashing [13]. Additionally, this method proposed probabilistic K-nearest neighbor (PKNN) and hierarchical clustering 
workloads. However, the method used the expensive measure of dynamic time warping (DTW) computation for filtering. Time series data mining prediction is depicted in Fig. 1.

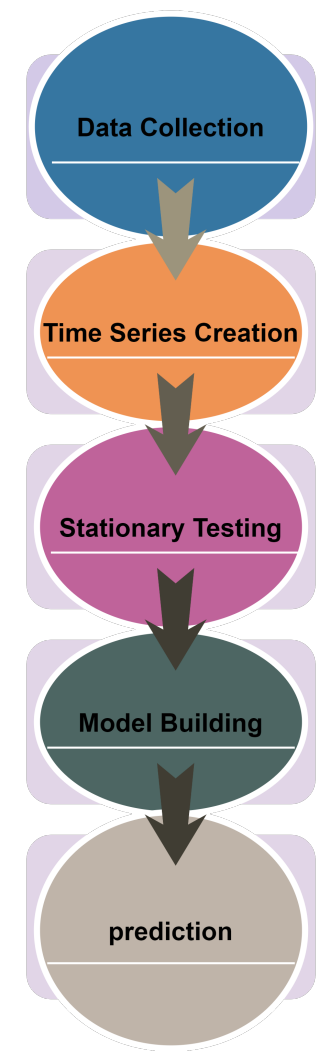

Fig. 1. Time series data mining prediction.

A principal component analysis-based algorithm has been introduced for similarity search on multivariate time series (MTS) data sets [14]. However, it does not reflect changes in the relationships between the variables. There has been relatively little progress in handling anomalies of similarities in time series data. Handling these anomalies requires a robust algorithm. Therefore, the proposed novel matrix profile (NMP) algorithm has the capability of precomputing and storing some information that can be used for data mining tasks, and the data mining process can thus be greatly accelerated.

The NMP calculates and stores effective and easy-to-access all-pair-similarity-search information, and this information can be used in a variety of data mining activities that range from well-defined tasks (e.g., motif discovery) to more open-ended tasks (e.g., representation learning).

\subsection{Research Problem Exploration}

All-pairs-similarity search is a critical problem when the data objects have nearest neighbors. Many algorithms have been proposed for identification in the text domain, collaborative filtering, clustering, community discovery, duplicate detection, and query refinement. However, all of the existing text processing algorithms have time-series data mining similarity problems. There has been relatively little development on all-pairs-similaritysearch time series subsequences. To handle this problem, state-of-the-art solutions have been proposed, which handle the following tasks in time series data prediction:

- Providing the full join method, which eliminates the need to specify a similarity threshold.

- $\quad$ Recovering the top-K nearest neighbors or the nearest neighbor for each object if that neighbor is within a user-supplied threshold. 
However, these solutions provide partial support for time series data in data mining. Thus, there is a need for a scalable algorithm to handle data mining tasks in time series to address large data sets and generate approximate solutions of higher quality in a reasonable amount of time.

\subsection{Research Significance}

The lack of consistency and interpretations in a business may cause the loss of business. This situation occurs when observations are not constant over time due to time series data. To address this issue, the NMP algorithm is introduced, which is compatible with large data sets and produces approximate solutions of higher quality in a short time. The significance of this work is to address several data mining tasks, such as motif discovery, discord discovery, long-term evolution (LTE) discovery, semantic segmentation, and clustering. By efficiently handling these tasks, business-related search activities can be greatly improved.

\subsection{Research Contribution}

The contributions of this article are summarized as follows:

- $\quad$ The NMP uses an ultrafast similarity search process based on the z-normalized Euclidean distance as a subroutine, exploiting the redundancies between overlapping subsequences to achieve dramatic speedup and low space overhead.

- $\quad$ The proposed NMP provides no false positives or false negatives. This property is important in many domains.

- $\quad$ The NMP provides lower space complexity, namely, $O(n)$, which helps in handling several data mining tasks (e.g., motif discovery, discord discovery, LTE discovery, semantic segmentation, and clustering).

\subsection{Proposed Solution}

A novel matrix profile algorithm is proposed to solve the all-pairs-similarity search problem for time series data. The proposed algorithm leverages the significant features from the STAMP [13] and STOMP [16] algorithms to provide a lower time for time series data search. The proposed NMP algorithm involves different similarity search measures (shape-based, edit-based, feature-based, and structure-based). The proposed method also consists of two algorithms, the distance determination process for neighboring nodes and immediate neighbor detection, which help to determine the similarity of the time series data.

\subsection{Research Structure}

The remaining parts of this paper are structured as follows: Section 2 includes the salient features of the related work. Section 3 describes the matrix profile (MP) plan of the research. Section 4 covers the implementation and results. Section 5 contains a discussion of the implementation, including its advantages and shortcomings. Finally, Section 6 concludes the paper and provides future analysis.

\section{Related Work}

In this section, the salient features of existing methods are extensively discussed. Wan and Davis [17] proposed an auto-distance covariance function for serial dependence evaluation to support the estimate residuals. The proposed method is based on time series model classification. The proposed method correctly identified misconfigurations when it was applied superfluously. However, the proposed model failed to justify the claimed idea. Furthermore, this process requires higher complexity.

Michael and Karsin [17] proposed several techniques for optimizing self-joining using a GPU, which included a GPU-efficient index that employs a bounded search, a batching scheme to accommodate large result sets, and duplicate search removal with low overhead. In addition, they proposed a performance model that reveals the bottlenecks related to the result of a given data set size and enables the selection of a batch size that mitigates 
two sources of performance degradation. However, the proposed model fails to calculate the distance between points, and it degrades the performance of index searches when the search distance is increased. Kalmykov LV and Kalmykov [18] exploited a similarity threshold more aggressively to limit the set of candidate pairs that are considered to reduce the amount of information indexed initially. The proposed method is based on the discrete Fourier transform (DFT). However, the method is complex, with many parameters to adjust. Yoon et al. [19] proposed an efficient method to detect large-scale multimedia data using waveform similarity that overcomes the disadvantages of existing detection methods. The proposed mechanism consists of an efficient supervised multimodal hashing method that focuses on directly employing semantic labels to monitor the hashing learning process.

Feng et al. [20] proposed the partition-based similarity search (PSS) algorithm, which uses a static partitioning algorithm that places dissimilar vectors into different groups and balances the comparison workload with a circular assignment. Wang et al. [21] proposed the label consistent matrix factorization hashing (LCMFH) algorithm, which maps heterogeneous information that focuses on an inert space and then adjusts the idle space to an inactive semantic space obtained from class labels. However, LCMFH does not consider the shortcomings of quantization, which corrupts the separation of hash codes. Johnson [22] proposed an algorithmic structure of similarity search methods that achieves near-optimal performance (NOP) on a graphical process that helps to perform close ideal execution on graphical processing units. However, the performance of these implementing algorithms is complex and counterintuitive.

Geng et al. [16] introduced STOMP for addressing large volumes of short text messages that lead to noise and redundancies. STOMP is particularly designed to deal with the microblogging stream on Facebook. STOMP also consists of a framework to handle time series data.

All of the existing proposed methods for time series are either complex or do not scale well for similarity search from a data mining perspective. Our proposed algorithm has less complexity and is highly compatible with similarity search from a data mining perspective.

\section{Proposed Novel Matrix Profile for Similarity Search}

The proposed NMP consists of three phases:

- $\quad$ Inputting the time series (ITS)

- $\quad$ All-pairs-similarity search process (ASSP)

- $\quad$ Distance determination \& matrix profile index (DD\&MPI)

\subsection{Inputting the Time Series (ITS)}

It performs collection of observations sequentially over time and occurs in a variety of Fields (e.g economics, and in financial, physical, marketing, demographic, engineering). These series can be univariate or multivariate, which is when several series simultaneously span multiple dimensions within the same time range. Fig. 2 depicts proposed model for time series data. It involves components that are common to most time series mining tasks.

\subsubsection{Data Preprocessing}

Issues of noise filtering, outlier handling, scale concerns, normalization and resampling of time series usually arise in time series in realistic situations. All of these problems are usually handled by preprocessing the data. Boris [23] proposed a fast algorithm for filtering noise for time series prediction using recurrent neural networks (RNNs). Li et al. [24] exploited the sliding window prediction (SWP) model to perform the correction of outliers. Additionally, they used standard temperature time series data gathered from the Nanjing University of Information Science and Technology (NUIST) weather stations.

The next concern relates to different variations in the scale of time series. As proposed by Pelletier et al. [25], a linear transformation of the amplitudes can handle this problem. Slawek [26] proposed a dynamic computational graph neural network (DCGNN) system, which received an award in the M4 competition. For the preprocessing, the time series 


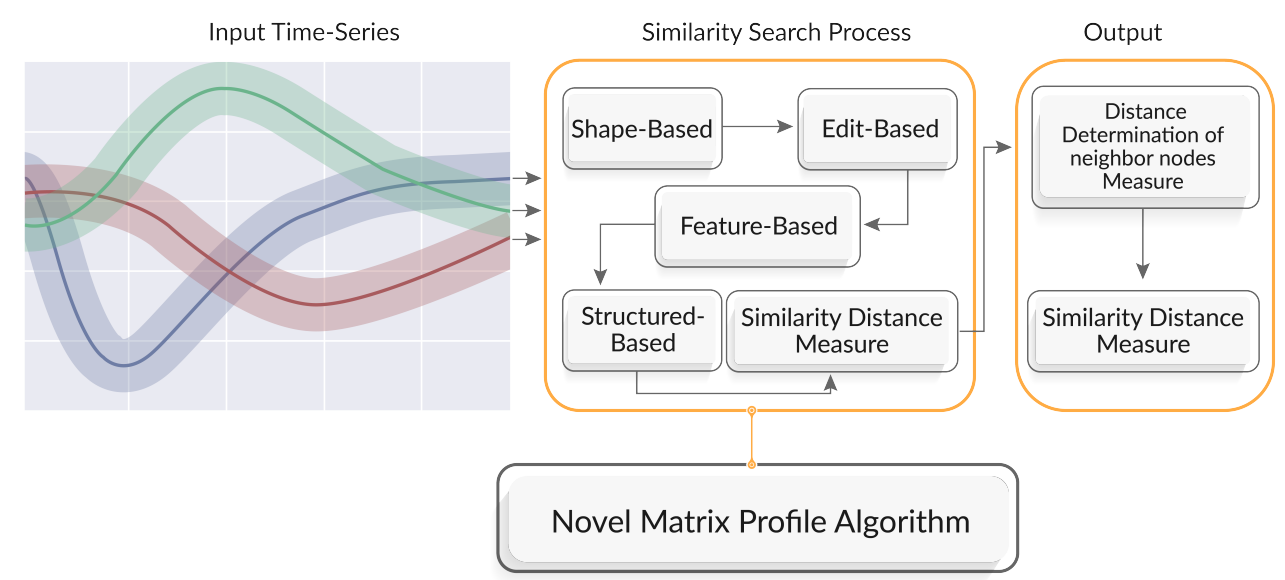

Fig. 2. Proposed model for time series data set supported with a NMP algorithm.

data were normalized by dividing them in the input window, which resulted in input and output values close to 1 .

Because data normalization is a fundamental preprocessing step, we will find an appropriate method to address time series normalization tasks. Here is one of the traditional data normalization methods:

Definition 1. A time series $T$ is called Z-normalized when its mean $\mu$ is 0 and its standard deviation $\sigma$ is 1 . The normalized version of $T=t_{1}, \ldots, t_{|T|}$ is computed as follows:

$$
c^{n}=\left\{\frac{t_{1}-\mu}{\sigma}, \ldots, \frac{t|T|-\mu}{\sigma}\right\}
$$

where $T^{n}$ is the $z$-based normalized time series of $T ; t_{1}, \cdots, t_{|T|}$ is a sequence of numbers $t_{i} \in \mathbb{R}$, where $i \in N$ represents the position in $T ;|T|$ is the length or size of time series $T$; and $\sigma$ is the standard deviation of time series $T$.

This approach works well in stationary settings where the exact minimum and maximum values of the time series are unknown, but it struggles with nonstationary time series where the mean and standard deviation change over time.

On the other hand, this method is useful when the minimum and maximum values of an attribute are undefined and can be extended to stationary time series, in other words, time series in which statistical properties such as the mean, variance, and autocorrelation remain constant over time.

Moreover, z-normalization is an essential operation in several applications because it allows similarity search irrespective of shifting and scaling.

\subsubsection{Data Representation}

Time-series data representation emphasizes the following essential characteristics:

- Temporal representation: storing a temporal point on the displayed data.

- Spectral representation: designing the data in the frequency domain.

- Other representations: implementing different modifications not related to the above.

This approach is described in a concise way and gains additional advantages, such as efficient storage, speedup of processing, and implicit noise removal. The essential characteristics of data representation require the following for any representation:

- A significant drop in the data dimensionality.

- An emphasis on the essential shape features for both global and local scales.

- Lower computational costs for the computational representation.

- Better restoration quality for the reduced representation. 
- Implicit noise handling or insensitivity to noise.

By studying several properties of a sequence at the same time, issues such as amplitude, scaling, temporal warping, noise, and outliers can be prevented. The following transformations are considered for a given time series $\mathrm{T}$ with $\mathrm{n}$ data points $T=\left\{t_{1}, \ldots, t_{n}\right\}$ : Amplitude shifting: The series $G=\left\{g_{1}, \ldots, g_{n}\right\}$ obtained by a linear amplitude shift of the original series is given by

$$
g_{i}=t_{i}+k
$$

where $g_{i}$ is the series of $T ; t_{i}$ is a time series such that $t_{i} \in T$; and $k$ is a constant, where $k \in \mathbb{R}$.

Uniform amplification: The series $G$ obtained by multiplying the amplitude of the original series is specified as:

$$
g_{i}=k \times t_{i}
$$

Uniform time scaling: The series $G=\left\{g_{1}, \ldots, g_{m}\right\}$ produced by a uniform change in the time scale of the original series is given as follows:

$$
g_{i}=t_{|k \times i|}
$$

Dynamic amplification: The series $G$ obtained by multiplying the original series by a dynamic amplification function that is assigned is:

$$
g_{i}=h(i) \times t_{i}
$$

where $h(i)$ is a function such that $\forall t \in[1,2, \ldots, n], h^{\prime}(t)=0$ if and only if $t_{i}^{\prime}=0$.

Dynamic time scaling: The series $G$ obtained by a dynamic change in the time scale is generated by

$$
g_{i}=t_{h(i)}
$$

where $h(i)$ is a positive, strictly increasing function such that $h$ is:

$$
\mathbb{N} \longrightarrow[1, \ldots, n]
$$

where $n$ is the length of $\mathbb{N} \in n$.

Additive Noise: The series $G$ obtained by adding a noisy component to the original series is given by

$$
g_{i}=t_{i}+\varepsilon_{i}
$$

where $t_{i}+\varepsilon_{i}$ and $\varepsilon_{i}$ are independent identically distributed white noise.

Outliers: The series $G$ is obtained by adding outliers at random positions. Formally, these are a given set of random time positions expressed by:

$$
\rho=\{k \in[1, \ldots, n]\}
$$

where $\rho$ is a random time position and $g_{k}=\varepsilon_{k}$, where $\varepsilon_{k}$ is independent identically distributed white noise.

Definition 2. The similarity measure $D(T, U)$ between time series $T$ and $U$ is a function that takes two time series as inputs and returns the distance d between the series. This distance has to be nonnegative; i.e., $D(T, U) \geq 0$.

If this measure satisfies the additional symmetry property $D(T, U)=D(U, T)$ and subadditivity $D(T, V) \leq D(T, U)+D(U, V)$ (also known as the triangle inequality), the distance is said to be a metric.

The similarity measure $D(T, G)$ should be robust to any combination of these transformations. This property leads to our formalization of four general types of robustness. We introduce properties that express robustness for scaling (amplitude modifications), 
warping (temporal modifications), noise and outliers. Let $S$ be a collection of time series, and let $H$ be the maximal group of homeomorphisms under which $S$ is closed.

A similarity measure $D$ on $S$ is called scale robust if it satisfies the following:

Property 1. For each $T \in S$ and $\alpha>0$, there is a $\delta>0$ such that $\left\|t_{i}-h\left(t_{i}\right)\right\|<\delta$ for all $t_{i} \in T$, which implies $D(T, h(T))<\alpha$ for all $h \in H$.

We call a similarity measure warp robust if the following holds:

Property 2. For each $T=\left\{t_{i}\right\} \in S, T^{\prime}=\left\{t_{h}(i)\right\}$ and $\alpha>0$, there is a $\delta>0$ such that $\|i-h(i)\|<\delta$ for all $t_{i} \in T$ implies that $D\left(T, T^{\prime}\right)<\alpha$ for all $h \in H$.

We call a similarity measure noise robust if it satisfies the following property:

Property 3. For each $T \in S$ and $\alpha>0$, there is a $\delta>0$ such that $U$ with $p(\varepsilon)=N(0, \delta)$ implies $(T, U)<\alpha$ for all $U \in S$.

$$
U=T+\varepsilon
$$

where $U$ is a function such that $U \in T$ and $\varepsilon$ is independent identically distributed white noise.

We call a measure outlier robust if the following holds:

Property 4. For each $T \in S, K=\{\operatorname{rand}[1, \ldots, n]\}$ and $\alpha>0$, there is a $\delta>0$ such that if $|K|<\delta$, $U_{(k \in K)}=\varepsilon_{k}$ and $U_{(k \notin K)}$, then $D(T, U)<\alpha$ for all $U \in S$.

$$
U_{k \notin K}=T_{k}
$$

where $U_{k \notin K}$ implies $U_{k \in K}=\varepsilon_{k}$.

\subsection{All-Pairs Similarity Search Process}

It defines how any pair of time series is distinguished or matched and how an intuitive distance between two series is formalized. This measure should establish a notion of similarity based on perceptual criteria, thus allowing the recognition of perceptually similar objects even when they are not mathematically identical. Additionally, it searches for similarities in data objects. Every time-series mining task requires a subtle notion of similarity between time series that is based on the more intuitive notion of shape.

It involves different types of similarity search measures:

- $\quad$ Shape-Based

- Edit-Based

- Feature-Based

- $\quad$ Structure-Based

\subsubsection{Shape-Based Similarity}

Shape-based distances compare the overall shape of the series. On the other hand, dynamic time warping (DTW) provides a grasp of the local biases of the time axis by using nonuniform time warping as described by Hong et al. [27]. This measure is capable of matching different parts of a time series by allowing the time axis to be warped. The shortest warping path in a distance matrix determines the optimum alignment. A warping path is a set of contiguous matrix indices that describe the mapping of two time series. The optimal path minimizes the global warping expense, even though there is an exponential number of potential warping paths. DTW can be determined using dynamic time-complexity programming $O(n 2)$. The notion of the upper and lower envelopes is introduced in the approach proposed by Rubinstein and Zhao [28], which represents the maximum allowed warping technique, where the time complexity becomes $O(n)$. A temporal restriction can also be imposed on the duration of the DTW window. These strategies have been shown to 
increase not only the speed but also the level of accuracy, as they prevent extended warping from pathological matching.

These measures do not match any of the types of robustness. Even if the problems of scaling and noise can be handled in a preprocessing step, the warping and outlier issues must be addressed with more sophisticated techniques. The use of elastic measures can provide an elegant solution to both problems.

\subsubsection{Edit-Based Similarity}

The edit-based method is used to characterize the distance between two strings. The underlying idea is that the minimum number of operations needed to transform one string into another, with insertion, deletion and substitution, can represent the distance between strings.

The longest common subsequence (LCSS) algorithm is used to handle outliers or noisiness in matching two time series as discussed in Vishwakarma et al. [29]. For point matching and a warping threshold $\delta$, the LCSS distance utilizes the threshold parameter $\varepsilon$. To compare the shapes of subsequences between two series, the NMP utilizes the z-normalized Euclidean distance.

Property 5. The z-normalized Euclidean distance between two sequences of length $m$ is, in effect, a function of the correlation between the two sequences as given below:

$$
d_{x, y}=\sqrt{2 m(1-\operatorname{corr}(x, y))}
$$

where $d$ is the z-normalized Euclidean distance between two sequences $x$ and $y, m$ is the length of the two sequences $x, y$ and corr () is the Pearson correlation coefficient between the two sequences $x, y$.

Proof. First, we illustrate the following properties of the inner product of the z-normalized sequence:

$$
\sigma_{x_{i}^{m}\left(x_{i}-\mu x\right)^{2}}^{2}
$$

where $\sigma_{x}^{2}$ is the variance of the sequence $x$.

$$
m=\sum_{i}^{m}\left(\frac{x_{i}-\mu x}{\sigma x}\right)^{2}
$$

Using this, we can obtain the following equality:

$$
\begin{aligned}
& d(x, y)^{2}=\sum_{i}^{m}\left(\frac{x_{i}-\mu x}{\sigma x}-\frac{y_{i}-\mu y}{\sigma y}\right)^{2} \\
& =\sum_{i}^{m}\left(\frac{x-\mu x}{\sigma x}\right)^{2}+\sum_{i}^{m}\left(\frac{y-\mu y}{\sigma y}\right)^{2} \\
& -2 \sum_{i}^{m}\left(\frac{x-\mu x}{\sigma x}\right)\left(\frac{y-\mu y}{\sigma y}\right) \\
& =2 m\left(1-\frac{1}{m} \sum_{i}^{m}\left(\frac{x-\mu x}{\sigma x}\right)\left(\frac{y-\mu y}{\sigma y}\right)\right) \\
& =2 m(1-\operatorname{corr}(x, y))
\end{aligned}
$$

The Pearson correlation coefficient (PCC) calculates the strength of the linear association between two variables. Let $x$ and $y$ be random variables. The PCC is defined as

$$
\begin{aligned}
& \operatorname{corr} x, y=\frac{(E(x)-\mu x)(E(y)-\mu y)}{\sigma_{x y}} \\
& =\frac{\sum_{i=1}^{m} Q Q_{x, y}-m \mu_{x} \mu_{y}}{m \sigma_{x} \sigma_{y}}
\end{aligned}
$$


where $E$ is the expected distance between the two sequences $x$ and $y$ and $Q_{x, y}$ is the dot product of time series $T_{x, m}$ and $T_{y, m}$.

The proposed connection of the z-normalized Euclidean distance with the Pearson correlation coefficient is expressed by the following equation:

$$
\begin{aligned}
& d_{x, y}=\sqrt{2 m(1-\operatorname{corr}(x, y))} \\
& =\sqrt{2 m\left(1-\frac{Q_{x, y}-m \mu_{x} \mu_{y}}{m \sigma_{x} \sigma_{y}}\right)}
\end{aligned}
$$

Definition 3. The z-normalized Euclidean distance $d_{x, y}$ of two time series subsequences $T_{x, m}$ and $T_{y, m}$ can be evaluated as follows:

$$
d_{x, y}=\sqrt{2 m\left(1-\frac{Q_{x}, y-m \mu_{x} \mu_{y}}{m \sigma_{x} \sigma_{y}}\right)}
$$

where $\mu_{x}$ is the mean of $T_{x, m}, \mu_{y}$ is the mean of $T_{y, m}, \sigma_{x}$ is the standard deviation of $T_{x, m}$ and $\sigma_{y}$ is the standard deviation of $T_{y, m}$.

Considering that the correlation is limited to the range $[1,1], d_{x, y}$ can fall within the range $[0,2 \sqrt{m}]$ between two sequences of length $m$, where zero implies an ideal match and $2 \sqrt{m}$ corresponds to the worst possible match. As a consequence, the upper bound of $2 \sqrt{m}$ can be used to normalize distances to the range $[0,1]$, which can help us to equate matches of different lengths and enable us to identify and repeat thresholds to define degrees of resemblance by using $d_{x, y}$. Rather than defining a threshold that is dependent on $m$, we can define a more uniform similarity threshold for sequences of any length in this way. Linardi et al. [30] found that the normalization factor $\sqrt{m}$ could compare matches of different lengths, but no reference to the underlying mathematics was made. The distance bounds for $Z_{e d}$ of 0 and $2 \sqrt{m}$ lead to 1 and -1 correlation coefficients, respectively. This approach assumes that $d_{x, y}=0$ and $d_{x, z}=2 \sqrt{m}$ for any sequence $x$ of length $m$ with $\sigma_{x} \neq 0$. In the case of

$$
\begin{aligned}
& y=a x+b \\
& z=-a x+b
\end{aligned}
$$

where $z$ is the sequence of time series, $a, b$ are any values, for $a>0$.

Let us assume that we have sequence $s \in R^{m}$ and two noise sequences $n \in R^{m}$ and $n^{\prime} \in R^{m}$ that are sampled out of a normal distribution $N\left(0, \sigma_{N}^{2}\right)$. Then, the estimated distance between the two sequences obtained by applying the noise to the base sequence can be represented as follows:

$$
x=y=s+n
$$

where $s$ is an unknown constant for $x$ and $y$ and $n$ is a normal distribution, where $n \sim$ $N\left(0, \sigma_{N}^{2}\right)$.

$$
\mathbb{E}\left[d_{x, y}^{2}\right]=(2 m+2) \frac{\sigma_{N}^{2}}{\sigma_{S}^{2}+\sigma_{N}^{2}}
$$

where $\sigma_{N}^{2}$ is the variance of the noise and $\sigma_{S}^{2}+\sigma_{N}^{2}$ is the predicted variance of the noisy sequence. 
We now determine the estimated effect of the noise. Henceforth, we assume sequences to be random variables and illustrate this idea by denoting them as $x$ and $y$.

$$
\begin{aligned}
& \mathbb{E}\left[d_{x, y}^{2}\right]=\mathbb{E}\left[\left(x_{1}-y_{1}\right)^{2}+\ldots+\left(x_{m}-y_{m}\right)^{2}\right] \\
& =m \cdot \mathbb{E}\left[(x-y)^{2}\right] \\
& =m \cdot \mathbb{E}\left[\left(\frac{x-\mu x}{\sigma_{x}}-\frac{y-\mu y}{\sigma_{y}}\right)^{2}\right]
\end{aligned}
$$

Since $x$ and $y$ are the products of identical variables, the two variables have equal variance.

$$
\sigma_{x}^{2}=\sigma_{y}^{2}=\sigma_{S}^{2}=\sigma_{N}^{2}
$$

Next, we decompose the additives $\mu_{x}$ and $\mu_{y}$ from the initial sequences $\mu_{s}$ and the noise effect. Here, we use $\mathrm{n}$ as a random variable from the noise distribution. We observe that $\mu S$ can be used as a constant by connection with the mean of the base sequence.

$$
\begin{aligned}
& \mu_{x}=\mu_{y}=\mu_{s}+\frac{n_{1}+\ldots+n_{m}}{m} \\
& =\mu_{s}+\mu_{n} \\
& \mu_{N} \sim N\left(0, \frac{\sigma_{N}^{2}}{m}\right)
\end{aligned}
$$

We perform the same decomposition for $x$ and $y$, where $s$ is an undefined constant from the base sequence:

$$
\begin{aligned}
& x=y=s+n \\
& n \sim N\left(0, \sigma_{N}^{2}\right)
\end{aligned}
$$

The constant expressions are cancelled, and the distributions are merged, yielding the following:

$$
\begin{aligned}
& \mathbb{E}\left[d_{x, y}{ }^{2}\right]=m \cdot \mathbb{E}\left[\left(\frac{n_{x}-n_{y}-\mu N_{x}+\mu N_{y}}{\sqrt{\sigma_{S}^{2}+\sigma_{N}^{2}}}\right)^{2}\right] \\
& m \cdot \mathbb{E}\left[\left(V^{2}\right)\right] \\
& V \sim N\left(0 \cdot \frac{2+2 m}{m} \cdot \frac{\sigma_{N}^{2}}{\sigma_{S}^{2}+\sigma_{N}^{2}}\right)
\end{aligned}
$$

Finally, we apply the theorem $\mathbb{E}[x]^{2}=\operatorname{var}(x)+\mathbb{E}[x]^{2}$ :

$$
\mathbb{E}\left[d_{x, y^{2}}\right]=(2 m+2) \cdot \frac{\sigma_{N}^{2}}{\sigma_{S}^{2}+\sigma_{N}^{2}}
$$

Theorem 1. Given a time series, the position that gives the maximum average point-to-point distance is the position that has the highest absolute Z-normalized value.

Proof. After Z-normalization, the mean and standard derivation of a time series are 0 and 1 , respectively. For any fixed point $a$, the expected distance between this factor and other factors is

$$
\begin{aligned}
& E\left[(X-a)^{2}\right]=E\left[X^{2}-2 X a+a^{2}\right] \\
& =E\left[X^{2}\right]-2 a E[X]+a^{2} E[1] \\
& =E\left[(X-\mu)^{2}\right]-2 a \mu+a^{2} \\
& =\operatorname{var}(X)-2 a \mu+a^{2}=1+a^{2}
\end{aligned}
$$

where $E$ is the expected point-to-point distance $a \geq E \geq 1+a^{2}, a$ is any fixed point, and $X$ is the expected maximum value. Consequently, the average point-to-point distance from a fixed point a to different points is $1+a^{2}$. Therefore, the point with the highest absolute Z-normalized value can be the predicted point-to-point distance. 
Lemma 1. The optimal ordering for early abandonment of the Euclidean distance is ordering by the absolute Z-normalized value.

Proof. An outline of all point-to-point distances is the Euclidean distance, and it is a monotonically nonreducing function. From Theorem 1, the predicted contribution to the summation can be increasingly expanded to large amounts as viable if we order the points in the series by their absolute Z-normalized values for each dimension. The sum of points used for early abandonment is then minimized, and the result determines the optimum order. In line with Lemma 1, we can obtain a perfect order in early abandonment by ordering the absolute Z-normalized values. Next, we can empirically enumerate the number of point-to-point predictions in the early abandonment process.

\subsubsection{Feature-Based Similarity}

Feature-based distances extract features that describe aspects of the series that are then compared with any type of distance function. This measure is mostly used to determine the local similarities between patterns. However, when handling longer time series, it might be profitable to find similarities on a global scale. This approach has the following properties:

- It uses an ultrafast similarity search process and the z-normalized Euclidean distance as a subroutine.

- It exploits the redundancies between overlapping subsequences.

- It provides lower space complexity to handle several data mining tasks.

\subsubsection{Structure-Based Similarity}

The main purpose of structure-based similarity is to find higher-level structures in the series. This approach compares structures on a global scale. It is divided into two further subcategories:

- Model-based distances.

- Compression-based distances.

1. Model-based distances

This approach applies a model to different series and then compares the parameters of the underlying model. Similarity can be calculated by modeling the underlying time series. This determines the probability of one time series by using another underlying model. Any type of parametric temporal model can be used. Furthermore, the distance determination process for neighboring nodes is given in Algorithm 1.

In Algorithm 1, the distance of the neighbor node is determined. In step 1, the variables are initialized. The input and output are given at the beginning of the algorithm. In step 2, the time series length is detected from the time series data. Steps 3-4 define the memory allocation process, and the initial matrix profile and matrix profile index are stored in memory. Steps 5-7 calculate the mean value and standard deviation. Step 8 is used to perform the vector dot product. In step 9, the distance profile index is determined from the time series. In steps 2-13, the distance of each neighbor is calculated and finally stored in an array.

2. Compression-based distances

Compression-based methods determine how easily two series can be compacted together. One distance measure is based on Kolmogorov complexity, called the compression-based dissimilarity measure (CDM). It is established based on bioinformatics findings. The fundamental notion is that concatenating and compressing similar series produces higher compression ratios for different data. If this method is successful for clustering, then it can be extended for fetal heart rate tracing. This process is effective for clustering. 


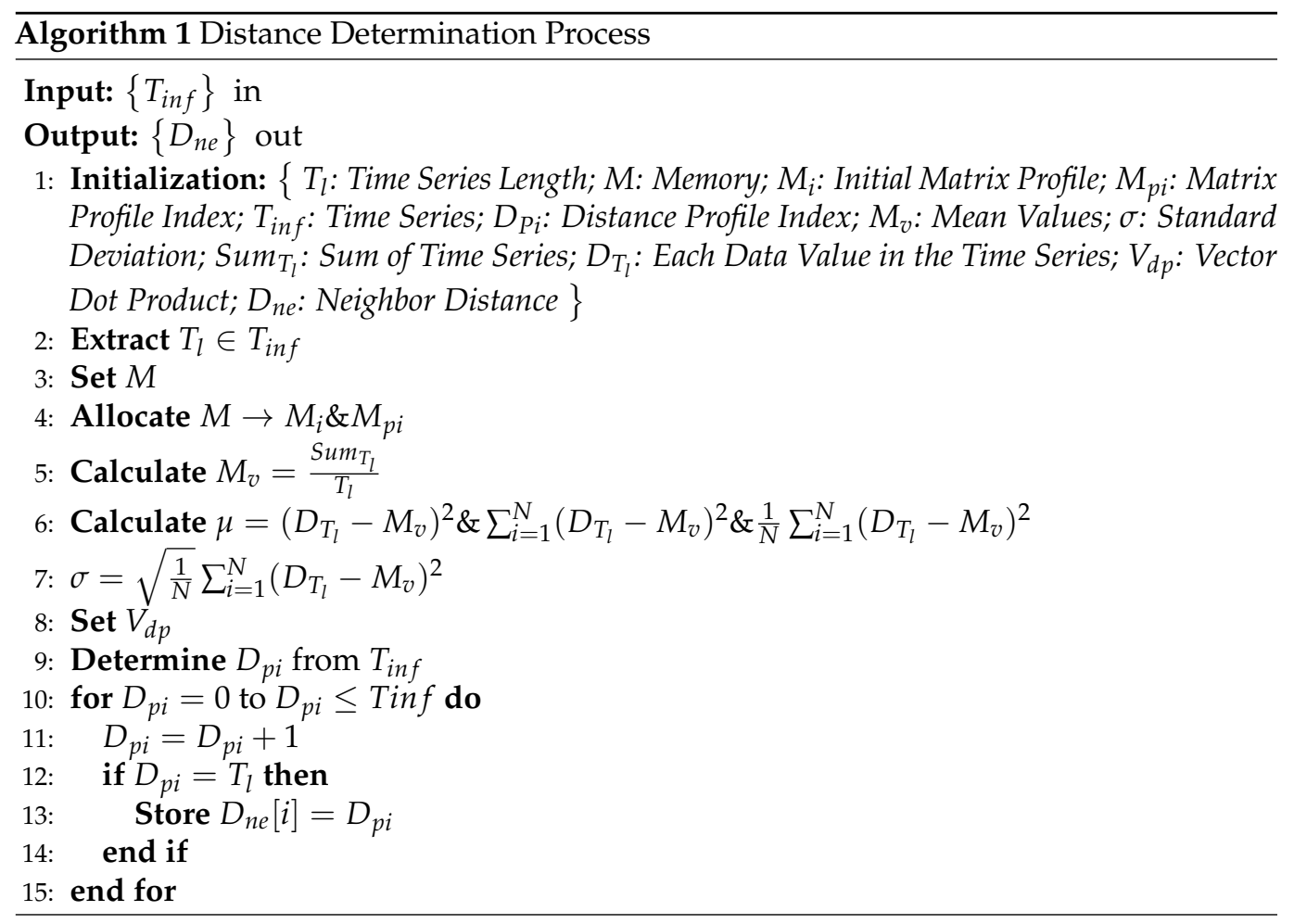

Our basic approach is to find similar pairs to compute the dot product of each normalized time series over the z-normalized Euclidean distance $d_{x, y}$. In other words, the dot product $\varrho_{i, j}$ can be evaluated in $O(1)$ when $\varrho_{i-1, j-1}$ is given:

$$
\varrho_{i, j}=\varrho_{i-1, j-1}-t_{i-1} t_{j-1}+t i+m-1 t_{j+m-1}
$$

where $\varrho_{i, j}$ is the dot product of $T_{i, m}$ and $T_{j, m} ; \varrho_{i-1, j-1}$ implies $Q_{i, j} \in t_{i, j}$, where $i, j \in m$; and $t_{i-1} t_{j-1}$ implies $t \in T$, where $i, j \in m$.

Before finding the distance profile, we search for similarities, where each normalization of each subsequence must be normalized before it is compared to the query by defining the mean value and the standard deviation. The mean of the subsequence can be calculated by holding two running sums of a long time series with a lag of exactly $\mathrm{m}$ values. Similarly, the sum of the subsequence squares can be determined. Here, consistency can be determined by the following:

$$
\mu=\frac{1}{m} \sqrt{x_{i}} .
$$

$m$ is the lag of the values taken by the sums of the long time series, and $x_{i}$ are the sums of the time series $T$.

The standard deviation of the time series calculated by the average of the squared deviations from the mean is shown below:

$$
\sigma^{2}=\frac{1}{m} \sum x_{i}^{2} \mu^{2}
$$

There is another name for them, "one pass". With the help of one pass measures, we will determine the distance profile $D_{P i}$.

Given a $T_{i, m}$ and a time series $T$ query list, the distance between $T_{i, m}$ and all subsequences is determined in $T$. We call this a distance profile: 


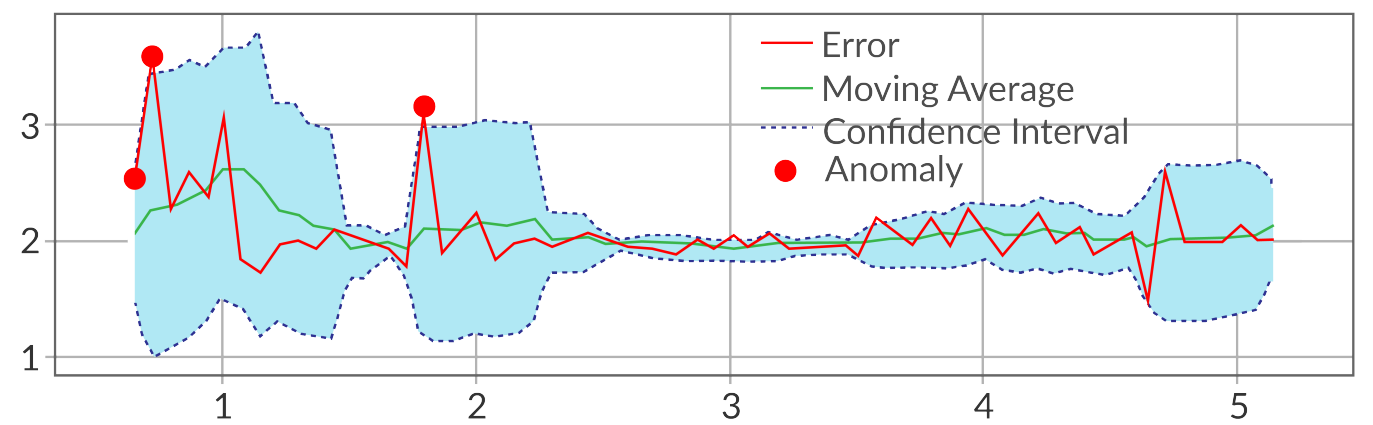

Fig. 3. Determination of confirmed outliers and anomalies for time series data sets

Definition 4. A distance profile $D_{p i}$ that corresponds to query $T_{i, m}$ and time series $T$ is a vector of the Euclidean distances between a given query subsequence $T_{i, m}$ and each subsequence in time series T. Formally,

$$
D_{i}=\left[d_{i, 1}, d_{i, 2}, \ldots, d_{i, n-m+1}\right]
$$

where $d_{i, j}(1 \geq j \geq n-m+1)$ is the distance between $T_{i, m}$ and $T_{j, m}$.

Once we have $D_{i}$, we will dispose of the closest neighbor to $T_{i, m}$ in time series $T$.

Note that if query $T_{i, m}$ is a subsequence of $T$, the i-th distance profile position $D_{i}$ is zero (i.e., $d_{i, i}=0$ ), and the value is near zero only to the left and right of $i$. In the literature, this match is considered a trivial match. We prevent such matches by ignoring the $m / 4$ duration "exclusion" place earlier and when $i$ is the location of the query. In practice, we set the following property:

$$
d_{i, j}\left(i-\frac{m}{4} \geq j \geq i+\frac{m}{4}\right) \in \infty
$$

where $\infty$ is the value of infinity where $d_{i, j}$ is appropriate.

Therefore, the nearest neighbor of $\left.T_{(} i, m\right)$ can be identified by evaluating $m$ in $\left(D_{i}\right)$.

\subsection{Distance Determination E Matrix Profile Index (DDEMPI)}

This index is the output value of the distance determination and index detection neighbor nodes. Indexing makes it possible for large-scale databases to provide an accessible organization of data for fast retrieval. Fig. 3 shows the three types of time-series data that are arranged according to their characteristics, but a few time-series data sets are out of reach of the neighbor nodes. Thus, such data sets are confirmed outliers and anomalies.

It is important to carefully describe the difference between time series data sets to show perceptually significant aspects of the underlying similarity. Finally, the indexing mechanism must allow ever-growing large data sets to be handled and queried effectively.

The immediate neighbor detection process is further described in Algorithm 2.

Algorithm 2 determines the immediate neighbor detection process. In step 1, the variables used in the algorithm are initialized. The input and output are shown at the beginning of the algorithm. In step 2, the length of the time series is calculated. In steps 3-4, we set and allocate the memory. In steps 5-7, we determine the mean values and the standard deviation of the time series. In steps 8-13, we calculate and save the location of each neighbor. The output of the algorithm is the matrix profile index.

In the basic method, we want to locate the nearest neighbor of every subsequence in $T$. The nearest neighbor information is stored in two meta-time series, the matrix profile and the matrix profile index.

As the name suggests, the matrix profile is a profile that stores the minimum Euclidean distance of every subset of one time series with respect to another (or itself, called self- 


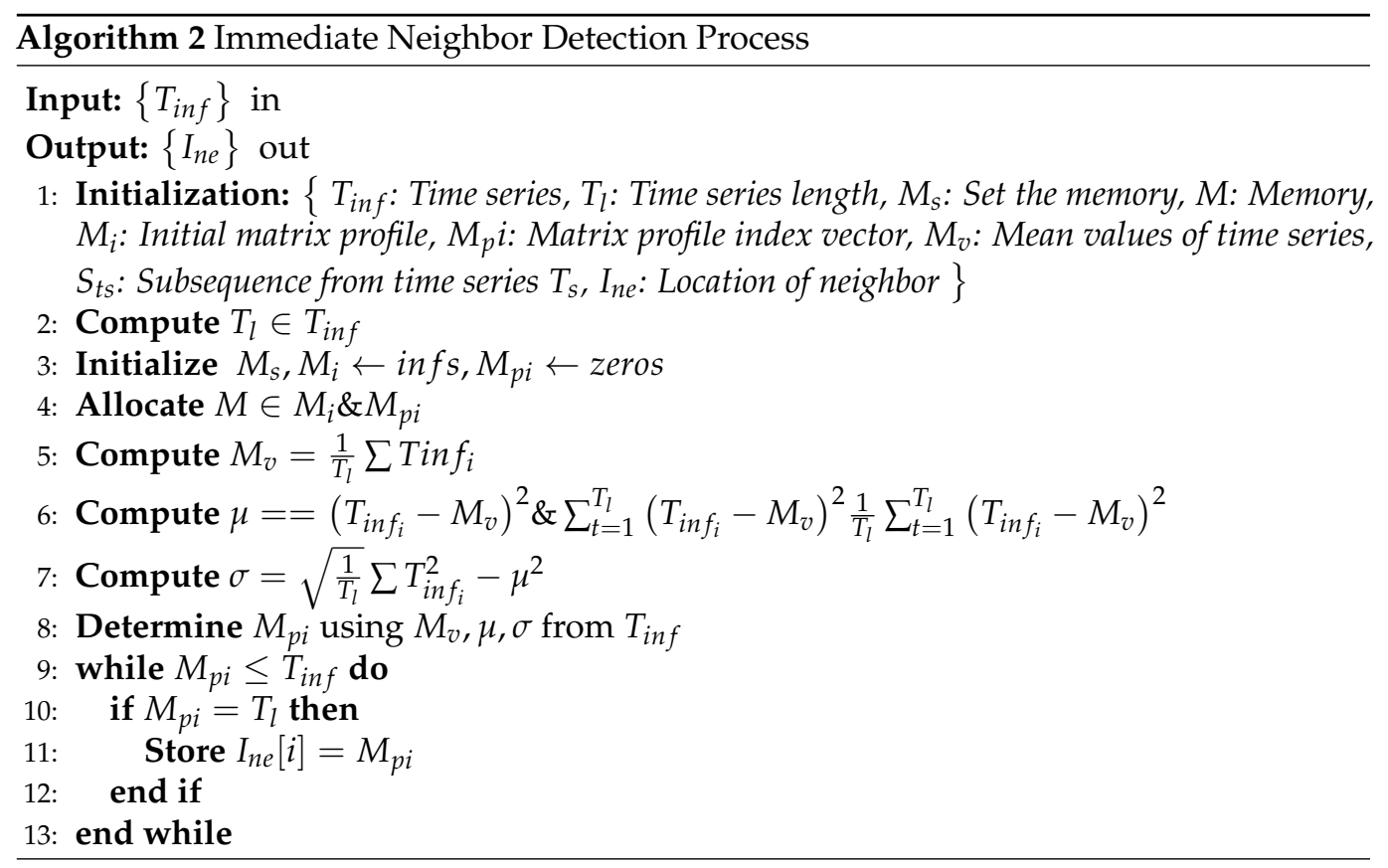

joining). It additionally stores an associate vector called the profile index that gives the index of each nearest neighbor.

The profile index $I_{n e}$ stores indices and therefore integers. The remaining meta-time series are all real values and are stored as floating point values. Overall, there are equations to define the values required for profile index $I_{n e}$, stored as floating point values:

$$
V_{f}=(n-m+1) \cdot 4+m-1
$$

where $V_{f}$ are floating point stored values, $n$ is the length of time series $T$ and $\mathrm{m}$ is the fixed length for each subsequence $T_{i, m}$. The next equation defines the values required for the remaining meta-time series of integer values:

$$
V_{i}=n-m+1
$$

where $V_{i}$ are stored integer values.

Dismissing the exclusion zone $E_{i}$ of trivial matches, the matrix profile denotes a vector whose elements are the minima of the columns in the distance matrix. The above proposed equations for computing the matrix profile are

$$
m_{p i}=\operatorname{mind}_{i, j}
$$

where $m_{p i}$ is the matrix profile.

The profile index $I_{n e}$ captures the starting index $j$ of this nearest neighbor. In the (theoretical) case of several minimizers $j$, the smallest minimizer will be selected:

$$
I_{n e}=\operatorname{argmind}_{i, j}
$$

where $I_{n e}$ is the profile index. Linardi et al. [30] proposed proofing next by using Parseval's theorem.

Theorem 2. The Euclidean distance between two signals $\vec{x}$ and $\vec{y}$ in the time series is the same as their Euclidean distance in the frequency domain. 
Proof. Let $\vec{X}$ be the discrete Fourier transform of the sequence $\vec{x}$. Then, we have

$$
\sum_{i=0, \ldots, n-1}\left|x_{t}\right|^{2}=\sum_{i=0, \ldots, n \times 1}\left|X_{f}\right|^{2}
$$

where $X_{f}$ is a linear map $f: X \rightarrow R_{k}$ and $x_{t}$ is a linear map $f: x \rightarrow R_{k+i}$.

The discrete Fourier transform inherits the properties below from the continuous Fourier transform. Let $\Leftrightarrow$ indicate Fourier pairs, i.e.,

$$
\left|x_{t}\right| \Leftrightarrow\left|X_{f}\right|
$$

where $\left|X_{f}\right|$ is the discrete Fourier transform of $\left|x_{t}\right|$. The discrete Fourier transform is a linear transformation: if the condition

$$
\left|x_{t}\right| \Leftrightarrow\left|X_{f}\right| ;\left|y_{t}\right| \Leftrightarrow\left|Y_{f}\right|
$$

holds, then we obtain the following:

$$
\left[x_{t}+y_{t}\right] \Leftrightarrow\left[X_{f}+Y_{f}\right]
$$

We then obtain

$$
\left[a x_{t}\right] \Leftrightarrow\left[a X_{f}\right]
$$

In addition, a shift in the time domain changes only the phase of the Fourier coefficients but not the amplitude.

$$
\left[x_{t-t_{0}}\right] \Leftrightarrow\left[X_{f} \exp \left(2 \pi f \frac{t_{0} j}{n}\right)\right]
$$

By the above equation, Parseval's theorem gives

$$
\|\vec{x}-\vec{y}\|^{2}=\|\vec{X}-\vec{Y}\|^{2}
$$

One of the aims of the next computation is to determine how much knowledge is lost because of the dimensionality reduction and time series reconstruction periods. We aim to determine how different the restored time series is from the original by using these equations.

The Euclidean distance between the predicted and real time series determines the reconstruction error of time series $T$ :

$$
\begin{aligned}
& \operatorname{Rec} \operatorname{Err}(T)=\sqrt{\sum_{i=1}^{n}\left(T_{t}-T^{\prime} t\right)^{2}} \\
& =\sqrt{\sum_{t=1}^{n}\left(T_{t}-(a \times t+b)\right)^{2}}
\end{aligned}
$$

where $T_{t}^{\prime}$ is the line segment $T_{t}^{\prime}=a \times t+b$ given for the well-approximated time series $T$ and where $a$ and $b$ are two coefficients that are a function of the linear curve.

These two parameters $a$ and $b$ satisfy the two criteria below:

$$
\begin{aligned}
& \frac{\partial \operatorname{Rec} \operatorname{Err}(T)}{\partial a}=0 \\
& \frac{\partial \operatorname{Rec} \operatorname{Err}(T)}{\partial b}=0
\end{aligned}
$$

By combining equations 43 and 44 , a and $\mathrm{b}$ can be obtained:

$$
a=\frac{12 \sum_{t=1}^{n}\left(t-\frac{n+1}{2}\right) T_{t}}{n(n+1)(n-1)}
$$




$$
b=\frac{6 \sum_{t=1}^{n}\left(t-\frac{2 n+1}{3}\right) T_{t}}{n(1-n)}
$$

where $T_{t}$ is the actual value at time stamp $t$ in time series $T$ and $a$ and $b$ are selected to achieve the minimum reconstruction error $T_{t}^{\prime}=a \cdot t+b$.

\section{Experimental Results}

In this section, we give the results of experiments regarding the experimental setup and performance metrics.

\subsection{Experimental Setup}

To confirm the performance of the proposed NMP algorithm, we use the Python platform to implement our algorithm. We compare our proposed NMP with the state-ofthe-art algorithms STAMP and STOMP. Table 1 shows the hardware and software used to implement the proposed algorithm.

Table 1. Development environment.

\begin{tabular}{cl}
\hline Parameters & Description \\
\hline Personal computer & x64 \\
Operating system & Windows 7 \\
Processor & Intel Core i7-2670QM \\
RAM & 6 GB \\
CPU GHz & 2.20 \\
\hline
\end{tabular}

Data sets. For the data sets, we used the single-dimensional time series and open data sources below. In the experimental investigations, we used real and synthetic data sets:

- $\quad$ Female birth: The total number of daily female births in California in 1959.

- ElectricProduction: Annual totals of electricity manufactured in California from 1985 to 2018.

- BeerProduction: Monthly Australian beer production data from 1956 to 1995.

- $\quad$ Random data: Three different data sets randomly generated with dates.

\subsection{Performance Metrics}

First, we should note that all of the implementations have been tested on common data sets of varying sizes. Here, there is no influence of NMP run-time performance on either data quality or the data inputs. To measure the algorithm's performance, we compared NMP with the STAMP and STOMP algorithms. Based on the testing results, the following metrics were considered:

- Time required for the similarity search

- Accuracy

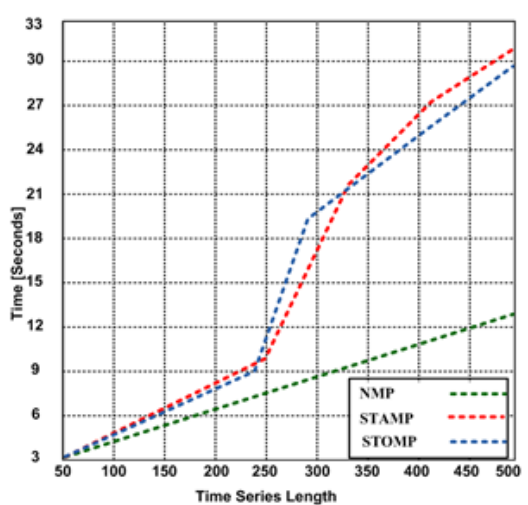

(a)

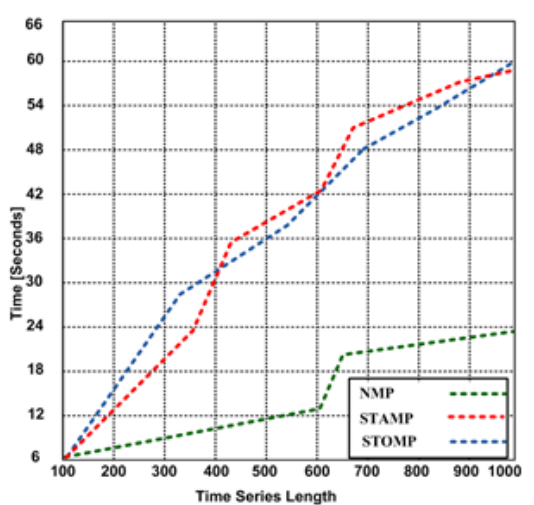

(b)

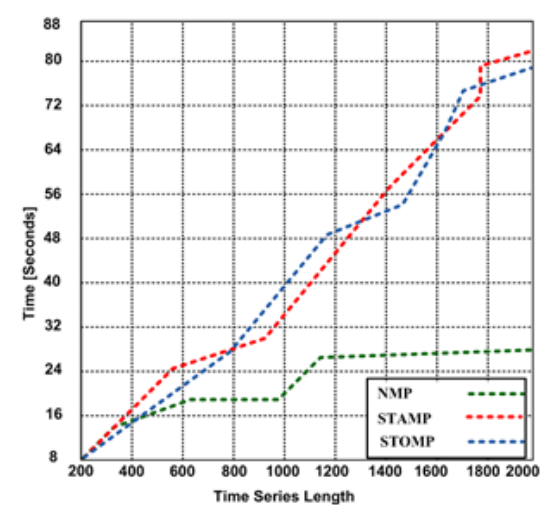

(c)

Fig. 4. (a) Run-time execution of NMP, STAMP and STOMP with a maximum query length of 500; (b) Run-time execution of NMP, STAMP and STOMP with a maximum query length of 2000; (c) Run-time execution of NMP, STAMP and STOMP with a maximum query length of 1000 . 


\subsubsection{Time Required for Similarity Search}

The run-time computation of a particular algorithm when performing the all-pairs similarity search is calculated as:

$$
T_{\text {req }}=\frac{T_{\text {cal }}}{n_{\text {cal }}^{2}} \times n_{\text {new }}^{2}
$$

where $T_{\text {req }}$ is the time spent for the performance computation of $T_{c a l}$ on every given hardware configuration, $T_{c a l}$ is the computing time in one calibration run, $n_{c a l}$ is the length of one calibration run and $n_{\text {new }}$ is the length of the time series $T$.

In Fig. 4a, we show the time required for the similarity search in the Female birth, ElectricProduction, and BeerProduction data sets. We generated the list of queries by attempting to extract random data series. Random time series data set queries of lengths 500, 1,000, 2,000 and 5000 were used. In Fig. 4a, we observe that STAMP and STOMP show similar times for time series query detection. Thus, it is observed that STAMP and STOMP have a certain time complexity in determining the length of the query. On the other hand, the proposed NMP appears to be compatible with the interval of the query. Based on the results, it is observed that NMP takes 12.9 seconds to detect the maximum 500 time series-length queries, whereas STOMP and STAMP take 30.05 seconds and 30.8 seconds, respectively.

In Fig. 4b, the maximum 1000 time series-length query is used. The result demonstrates that NMP takes 23.8 seconds to complete the 1000 time series-length query. However, the compared STAMP and STOMP take 59.1 and 60 seconds, respectively. It is also observed that the proposed NMP rapidly increases the time after 600 time series lengths. The compared STAMP and STOMP increase the time exponentially.

Fig. 4c shows that the proposed NMP takes 28 seconds to process the maximum 2000 time series-length queries, whereas the compared algorithms STOMP and STAMP take 79.1 and 81.9 seconds, respectively. The proposed NMP shows constant performance in time series detection after 1200 time series lengths. On the other hand, the compared algorithms increase the time exponentially, and the variable performance of the compared algorithms can affect the accuracy.

Fig. 5a shows that the proposed NMP takes 71.2 seconds to process the maximum 5000 time series-length queries, while the compared algorithms STOMP and STAMP take 154.2 and 160.9 seconds, respectively. The proposed NMP shows variable performance for time series detection. However, the proposed NMP takes less time than the compared algorithms. The compared algorithms increase the time exponentially despite having extended time series length.

\subsubsection{Accuracy}

The reconstruction accuracy of the time series is assessed. The main purpose of this experiment is to compute the amount of evidence loss, which is indicated by the results for the dimensionality reduction and time series reconstruction processes. The reconstruction accuracy depends on the Euclidean interval and the dimensionality of the time series. We can calculate it as a root-mean-square deviation:

$$
a_{r e q}\left(T, T^{\prime}\right)=\sqrt{\frac{\sum_{i=1}^{N}\left(t_{i}^{\prime}-t_{i}\right)^{2}}{N}},
$$

where $T$ is the original time series, $T^{\prime}$ is the time series reconstruction, $a_{r e c}$ is the reconstruction accuracy between $T$ and $T^{\prime}$ and $N$ is the dimensionality between time series $T$ and $T^{\prime}$.

The percentage of reconstructed accuracies can be seen in Fig. 5b. In addition, we increase the length of the time series to 10,000 to obtain exact plotting results. 


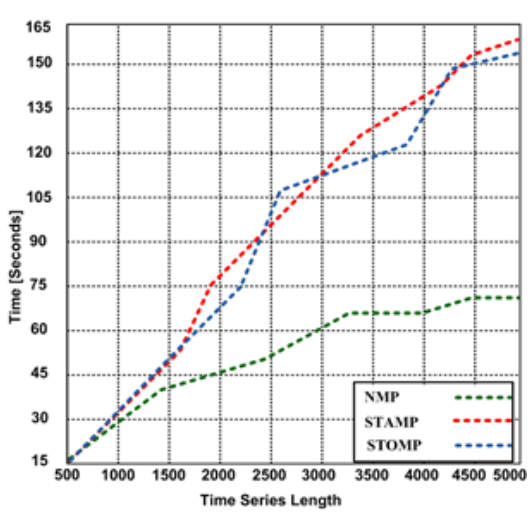

(a)

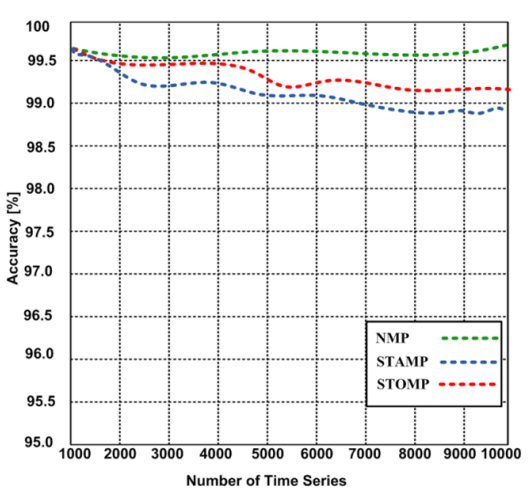

(b)

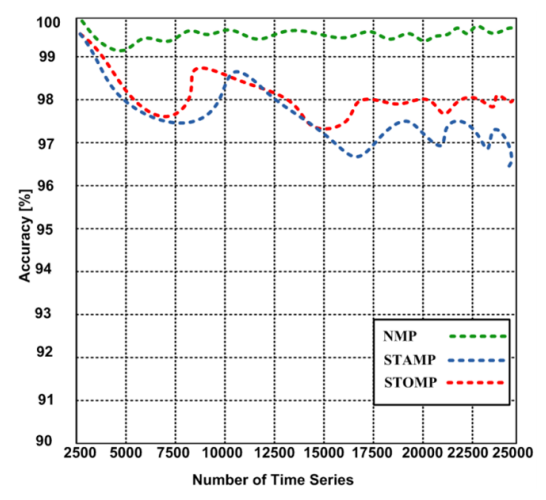

(c)

Fig. 5. (a) Run-time execution in seconds for NMP, STAMP and STOMP with $n=5000$, varying the time series length; (b) Accuracy of NMP, STAMP and STOMP with a maximum of 10000 for the time series data length; (c) Accuracy of NMP, STAMP and STOMP with a maximum of 25000 for the time series data length.

The results in Fig. 5a show that all representations have better results, but the NMP algorithm gives better results than the other algorithms. We observed that by increasing the length of the data, the accuracy of the STAMP and STOMP algorithms started to decrease. When NMP shows stable accuracy, it is found to be $99.63 \%$ with a maximum time series data length of 1,000. The compared STAMP and STOMP reduce the accuracy with an increase in the length of the time series. STAMP and STOMP show $98.95 \%$ and $99.23 \%$ accuracy, respectively.

In Fig. 5c, we observe that for a time series of length 25,000, the accuracy of all representations starts at $100 \%$. After increasing the data length to 25,000 , the accuracy of STAMP slows down to $96 \%$. Additionally, STOMP's reconstruction accuracy decreases to $98 \%$ when NMP shows an almost constant accuracy of $99.59 \%$.

\section{Discussion of the Results}

The proposed NMP possesses interesting properties to meet the requirements of matrix profile indexing. It is faster than the STAMP and STOMP algorithms and occupies less space compared to the other algorithms. The most important feature of the proposed NMP is that it handles queries of variable lengths. There is a very marginal chance of producing false positives or false negatives. With respect to the segmented representation, our matrix profile indexing is guaranteed to produce no false negatives. However, we cannot make the same claim with respect to the original results. Because such an occurrence involves pathological conditions and has never been found in any of our studies, we do not feel that this concern is a major limitation. Nevertheless, we will briefly address an extension to our representation that would allow us to change our indexing method to ensure no false negatives with regard to the original data. The data representation section can be extended from a 4-tuple to a 5-tuple, where the additional element is the residual error of approximating the rows. It is possible to adjust the distance measures stored in the index to provide a confidence bound, which is simply the sum of all the residual error terms from all of the segments that are compared. To determine the best segmented match for a query, an initial run of an indexing scheme can be used. The raw data can be indexed using this best fit, and the real Euclidean distance between the query and the best segmented raw data can be computed. Table 2 shows the performance of the proposed and compared algorithms.

\section{Conclusion and Future Work}

This section describes the achieved objectives and concludes the key findings for the reader. Additionally, it offers insight for future analysis. 
Table 2. The performance of the proposed and compared algorithms.

\begin{tabular}{cccl}
\hline Algorithm & STAMP & STOMP & NMP \\
\hline Time required for the similarity search with 500 time series & $30.8 \mathrm{~s}$ & $30.05 \mathrm{~s}$ & $12.9 \mathrm{~s}$ \\
Time required for the similarity search with 1,000 time series & $59.1 \mathrm{~s}$ & $60.0 \mathrm{~s}$ & $23.8 \mathrm{~s}$ \\
Time required for the similarity search with 2,000 time series & $81.9 \mathrm{~s}$ & $79.1 \mathrm{~s}$ & $28.0 \mathrm{~s}$ \\
Time required for the similarity search with 5,000 time series & $160.9 \mathrm{~s}$ & $154.2 \mathrm{~s}$ & $71.2 \mathrm{~s}$ \\
Accuracy with 10,000 time series & $98.95 \%$ & $99.23 \%$ & $99.63 \%$ \\
Accuracy with 25,000 time series & $96.0 \%$ & $98.0 \%$ & $99.59 \%$ \\
\hline
\end{tabular}

\subsection{Conclusion}

In conclusion, we propose a novel matrix profile algorithm for time series subsequence all-pairs similarity search. The proposed novel matrix profile is obtained in three phases. In the beginning, we preprocess and represent data sets by preventing time series issues such as amplitude, scaling, temporal warping, noise, and outliers. Then, we go through the distance determination process for neighboring nodes using the $\mathrm{z}$-normalized Euclidean distance method. The last phase consists of the immediate neighbor detection process used to determine the values required for the matrix profile index. The NMP has good qualities such as simplicity, high speed, parallelizability and a lack of parameters. Another advantage is its accuracy. The last expression is used to calculate the accuracy values. The accuracy of using NMP is $99.59 \%$ with a maximum time series length. Additionally, the run-time execution for the NMP algorithm is computed, and the time required for the novel matrix profile is compared to that of other existing state-of-the-art algorithms. The results show that the proposed NMP is the best choice for solving the all-pairs similarity search problem. Additionally, we show that our algorithm has implications for many current tasks, such as motif discovery, discord discovery, shapelet discovery and semantic segmentation, and new pathways can be opened up for science, including computing various definitions of time series set differences.

\subsection{Future Work}

We aim to focus on the security of the proposed algorithm, particularly false positives and false negatives, for the future. Additionally, vulnerabilities and potential attacks that could affect performance will be explored. Different quality-of-service metrics will also be investigated to determine the effectiveness of the proposed algorithm.

Author Contributions: A.R., conceptualization, writing, idea proposal, methodology, and results; M.A., data curation, software development, submission, and preparation; M.A. and B.A., review, manuscript preparation, and visualization; H. A., S. H. and A. A. review and editing. All authors have read and agreed to this version of the manuscript.

Funding: This research received no external funding.

Institutional Review Board Statement: Not applicable.

Informed Consent Statement: Not applicable.

Data Availability Statement: The data that supports the findings of this research is publicly available as indicated in the references.

Conflicts of Interest: The authors declare no conflict of interest.

\section{Appendix A. Definitions}

Definition A1. A time series $T$ is a sequence of real-valued numbers $t_{i}$ :

$$
T=t_{1}, t_{2}, \ldots, t_{n}
$$

where $n$ is the length of $T$. 
Typically, we are interested in a time series, and we are interested not in global but local properties. A local area of a time series is referred to as a subsequence:

Definition A2. A subsequence $T_{i, m}$ of a time series $T$ is a continuous subset of the values from $T$ of length $m$ starting at position i. Formally,

$$
T_{i, m}=t_{i}, d t_{i+1}, \ldots, t_{i+m-1}
$$

where $i$ is the index of time series $T$, where $1 \leq i \leq m-1$, and $d$ is the distance, where $d \ll m-1$.

Here, we can show that if we order the distance measurements for a given time series query based on the absolute values of the Z-normalized values, the average cumulative squared distance will be maximized. In other words, it is the right order in the general or in the normal case.

Definition A3. An all-subsequences set $A$ of a time series $T$ is an ordered set of all possible subsequences of $T$ obtained by sliding $a$ window of length $m$ across $T$ :

$$
A=\left\{T_{1, m}, T_{2, m}, \ldots, T_{n-m+1, m}\right\}
$$

where $m$ is a user-defined subsequence length. We use $A[i]$ to denote $T_{i, m}$.

Definition A4. 1NN-join function: given two all-subsequences sets $A$ and $B$ and two subsequences $A[i]$ and $B[j]$, a $1 N N-j o i n$ function $\theta 1 n n(A[i], B[j])$ is a Boolean function that returns "true" only if $B[j]$ is the nearest neighbor of $A[i]$ in set $B$. With the defined join function, a similarity join set can be generated by applying the similarity join operator on two input all-subsequences sets.

Definition A5. A self-similarity join set $J_{A A}$ is the result of a similarity join of the set $A$ with itself. We denote this formally as

$$
J_{A A}=A \bowtie_{\theta 1 n n} A,
$$

where $J_{A A}$ is the similarity join of sets $A[i]$ and $A[j]$. that

Two sequences $X$ and $Y$ are similar if they have long common subsequences $X^{\prime}$ and $Y^{\prime}$ such

$$
Y^{\prime} \approx a X^{\prime}+b
$$

where $a, b$ are constants or coefficients that are real numbers, $a_{1}, \ldots, a_{n} \neq 0$ and $b_{1}, \ldots, b_{n} \neq 0$. The overall similarity measure maximizes the above expression over all possible transformations $f$, and thus, we have

$$
\operatorname{Sim}(X, Y)=\max _{\text {allf }}\left\{\operatorname{Sim}_{f}(X, Y)\right\}
$$

where $\operatorname{Sim}(X, Y)$ is the similarity between sequences $X$ and $Y$; $\max _{\text {all }}$ is the local maximum of the first-order landmarks, where $\operatorname{Sim}_{f}(X, Y)$ is the similarity between the sequences

$$
X=x_{1}, x_{2}, \ldots, x_{n} \text {, and } Y=y_{1}, y_{2}, \ldots, y_{n} .
$$

Definition A6. Similarity join set: Given all-subsequences sets $A$ and $B$, a similarity join set $J_{A B}$ of $A$ and $B$ is a set that contains pairs of each subsequence in $A$ with its nearest neighbor in $B$ :

$$
J_{A B}=\left\{\langle A[i], B[j]\rangle \mid \theta_{1 N N}(A[i], B[j])\right\},
$$

where $J_{A B}$ is a similarity join set between $A$ and $B ; A[i], B[j]$ are subsequences from the same all-subsequences set $A$. We denote this formally as

$$
J_{A B}=A \bowtie_{\theta 1 N N} B,
$$

where $\bowtie_{\theta 1 N N}$ is a $1 N N$-join function of sets $A$ and $B$ such that $J_{A B} \neq J_{B A}$. 
Definition A7. A matrix profile P of time series $T$ is a vector of the Euclidean distances between every subsequence of $T$ and its nearest neighbor in T. Formally,

$$
\begin{gathered}
P=\left[\min \left(D_{1}\right), \min \left(D_{2}\right), \ldots, \min \left(D_{n-m+1}\right)\right], \\
P=\min ([M P ; d]),
\end{gathered}
$$

where $D_{i}(1 \leq i \leq n-m+1)$ is the distance profile $D_{i}$ that corresponds to query $T_{i, m)}$ and time series $T$.

Definition A8. A matrix profile index I of time series $T$ is a vector of integers:

$$
I=\left[I_{1}, I_{2}, \ldots, I_{n-m+1}\right]
$$

where $I$ is the index in $I_{i}=j$ if $d_{i, j}=\min \left(D_{i}\right)$.

Definition A9. A time series motif is the most similar subsequence pair of a time series. Formally, $T_{a, m} T_{b, m}$ is a motif pair if

$$
\operatorname{dis}\left(T_{a, m} T_{b, m}\right) \leq \operatorname{dis}\left(T_{i, m}, T_{j, m}\right) \in[1,2, \ldots, n-m+1],
$$

where $a \neq b, i \neq j$, and dist is a function that computes the z-normalized Euclidean distance between the input subsequences.

\section{References}

1. Li, H. Time works well: Dynamic time warping based on time weighting for time series data mining. Information Sciences 2021, 547, 592-608.

2. Sattari, M. T.; Avram, A.; Apaydin, H.; Matei, O. Soil Temperature Estimation with Meteorological Parameters by Using Tree-Based Hybrid Data Mining Models. Mathematics 2020, 8(9), 1407.

3. Zhang, S. Q.; Zhou, Z. H. Harmonic recurrent process for time series forecasting. In European Conference on Artificial Intelligence 2020, pp. 1714-1721. IOS Press.

4. Soleimani, G.; Abessi, M. DLCSS: A new similarity measure for time series data mining. Engineering Applications of Artificial Intelligence 2020, 92, 103664.

5. Gharghabi, S.; Ding, Y.; Yeh, C. C. M.; Kamgar, K.; Ulanova, L.; Keogh, E. Matrix profile VIII: domain agnostic online semantic segmentation at superhuman performance levels. In 2017 IEEE international conference on data mining (ICDM) 2017, November (pp. 117-126). IEEE.

6. Gharghabi, S.; Yeh, C. C. M.; Ding, Y.; Ding, W.; Hibbing, P.; LaMunion, S.; Keogh, E. Domain agnostic online semantic segmentation for multi-dimensional time series. Data mining and knowledge discovery 2019, 33(1), 96-130.

7. Guigou, F.; Collet, P.; Parrend, P. SCHEDA: Lightweight euclidean-like heuristics for anomaly detection in periodic time series. Applied Soft Computing 2019, 82, 105594.

8. Hu, M.; Feng, X.; Ji, Z.; Yan, K.; Zhou, S. A novel computational approach for discord search with local recurrence rates in multivariate time series. Information Sciences 2019, 477, 220-233.

9. Zhou, Y.; Ren, H.; Li, Z.; Pedrycz, W. An anomaly detection framework for time series data: An interval-based approach. Knowledge-Based Systems 2021, 107153.

10. Li, J.; Izakian, H.; Pedrycz, W.; Jamal, I. Clustering-based anomaly detection in multivariate time series data. Applied Soft Computing 2021, 100, 106919.

11. Crnkić, A.; Ivanović, I.; Jaćimović, V.; Mijajlović, N. Swarms on the 3-sphere for online clustering of multivariate time series and data streams. Future Generation Computer Systems 2020, 112, 11-17.

12. Yu, C.; Luo, L.; Chan, L. L. H.; Rakthanmanon, T.; Nutanong, S. A fast LSH-based similarity search method for multivariate time series. Information Sciences 2019, 476, 337-356.

13. Yang, K.; Shahabi, C. A PCA-based similarity measure for multivariate time series. In Proceedings of the 2 nd ACM international workshop on Multimedia databases 2004, November, pp. 65-74.

14. Wan, P.; Davis, R. A. Goodness-of-fit testing for time series models via distance covariance. Journal of Econometrics 2020.

15. Geng, F.; Liu, Q.; Zhang, P. A time-aware query-focused summarization of an evolving microblogging stream via sentence extraction. Digital Communications and Networks 2020, 6(3), 389-397.

16. Geng, F.; Liu, Q.; Zhang, P. A time-aware query-focused summarization of an evolving microblogging stream via sentence extraction. Digital Communications and Networks 2020, 6(3), 389-397. 
17. Gowanlock, M.; Karsin, B. Accelerating the similarity self-join using the GPU. Journal of parallel and distributed computing 2019, 133, 107-123.

18. Kalmykov, L. V.; Kalmykov, V. L. A solution to the dilemmalimiting similarity vs. limiting dissimilarity'by a method of transparent artificial intelligence. Chaos, Solitons \& Fractals 2021, 146, 110814.

19. Tsuchiyama, A.; Nakajima, J. Diversity of deep earthquakes with waveform similarity. Physics of the Earth and Planetary Interiors 2021, 314, 106695.

20. Wang, D.; Gao, X.; Wang, X.; He, L. Label consistent matrix factorization hashing for large-scale cross-modal similarity search. IEEE transactions on pattern analysis and machine intelligence 2018, 41(10), 2466-2479.

21. Johnson, J.; Douze, M.; Jégou, H. Billion-scale similarity search with gpus. IEEE Transactions on Big Data 2019.

22. Rubinstein, B. A fast noise filtering algorithm for time series prediction using recurrent neural networks. arXiv preprint arXiv:2007.080632020.

23. Ma, L.; Gu, X.; Wang, B. Correction of outliers in temperature time series based on sliding window prediction in meteorological sensor network. Information 2017, 8(2), 60.

24. Pelletier, C.; Webb, G. I.; Petitjean, F. Temporal convolutional neural network for the classification of satellite image time series. Remote Sensing 2019, 11(5), 523.

25. Smyl, S. A hybrid method of exponential smoothing and recurrent neural networks for time series forecasting. International Journal of Forecasting 2020, 36(1), 75-85.

26. Cai, X.; Xu, T. DTWNet: a Dynamic TimeWarping Network. Advances in Neural Information Processing Systems $2019,32$.

27. Hong, J. Y.; Park, S. H.; Baek, J. G. SSDTW: Shape segment dynamic time warping. Expert Systems with Applications 2020, 150, 113291.

28. Rubinstein, A.; Song, Z. Reducing approximate longest common subsequence to approximate edit distance. In Proceedings of the Fourteenth Annual ACM-SIAM Symposium on Discrete Algorithms 2020, pp. 1591-1600.

29. Vishwakarma, G. K.; Paul, C.; Elsawah, A. M. An algorithm for outlier detection in a time series model using backpropagation neural network. Journal of King Saud University-Science 2020, 32(8), 3328-3336.

30. Linardi, M.; Zhu, Y.; Palpanas, T.; Keogh, E. Matrix profile X: VALMOD-scalable discovery of variable-length motifs in data series. In Proceedings of the 2018 International Conference on Management of Data 2018, May, pp. 1053-1066. 